\title{
Investigating reservation policies of advance orders in the presence of heterogeneous demand
}

\author{
Bisheng Du, Christian Larsen* \\ Centre for Operations Research Applications in Logistics (CORAL), Department of Business Studies. \\ Aarhus School of Business, Aarhus University, Fuglesangs Allé 4, DK-8210 Aarhus V, Denmark
}

\begin{abstract}
We consider an inventory system, operated by a base stock policy and serving two customer classes. One customer class, Class 1, does not provide any advance demand information at all, while the other, Class 2, does. In order to reward a customer of Class 2 for providing advance order information, it may be sensible to make reservations for the order in the inventory system, thereby preventing later arriving orders from getting access to the inventory before this particular order. We propose various reservation policies and study their impact on the performance of the inventory system.
\end{abstract}

Keywords: Inventory control, advance demand information, heterogeneous demand

\section{Introduction}

We consider an inventory system serving two customer classes with different characteristics. The first class, in the following denoted Class 1, consists of customers who physically arrive at the inventory system and accordingly expect to get instantaneous delivery of their order requests. The second class, in the following denoted Class 2, does not physically arrive at the inventory system. Instead they submit their order requests for instance by email or via a webpage linked to the inventory system. They do not expect instantaneous delivery. Instead when submitting their order requests, they also specify a future time point when they want to receive their order. As an example, it is often the case that retailers, in addition to traditional sales from their shops, now also

\footnotetext{
*Corresponding author. Tel: +45 894 86317; Fax: +45 89486660

Email addresses: bisd@asb.dk (Bisheng Du), chl@asb.dk (Christian Larsen)
} 
provide sales via the Internet. Furthermore, entrepreneurs who start their business by organizing their sales from the Internet will often as their business grows to maturity, also start offering sales from shops. For a related case, see Simchi-Levi et al. (2008), pp. 201-202.

There are two interesting aspects of this example. The first is that of advance demand information. This means that when a request for an order occurs, say at time point $\tau$, the customer also specifies the time point, say time point $y+\tau$, when he wants to receive his order. Thus, $y$ can be interpreted as the demand lead-time of the customer. In many textbooks on inventory control, such as Zipkin (2000) and Silver et al. (1998), it is tacitly assumed that $y=0$. The first paper to make a systematic analysis of advance demand information in inventory control systems is Hariharan and Zipkin (1995). Here it is assumed that all customers have the same demand lead-time and that an improvement in performance corresponds to an equal reduction in the replenishment lead-time. A similar study by Gallego and Özer (2001) is based on a periodic review model where customers can have different demand lead-times. Here it is proven that a state dependent $(s, S)$ policy is optimal. Lu et al. (2003) and Marklund (2006) extend the model of Hariharan and Zipkin (1995) by studying multi-level systems: Lu et al. (2003) study an assembly system while Marklund (2006) studies a divergent distribution system. Related works are Chen (2001) investigating advance demand information and market segmentation and Tan (2008) analyzing the impact of advance demand information on forecast performance. For recent papers on advance demand information, see also Wang and Toktay (2008), Kunnumkal and Topaloglu (2008), Gayon et al. (2009), Tan et al. (2009) and Papier and Thonemann (2009). The second source of inspiration concerns the sort of inventory control policies which should be applied in the presence of heterogeneous demand, in particular whether a rule for how to prioritize orders from different customer classes should be incorporated. In the literature (see Topkis (1968), Evans (1968), Kaplan (1969), Frank et al. (2003) and Tempelmeier (2006) for periodic review models and Nahmias and Demmy (1981), Dekker et al. (1998), Dekker et al. (2002), Melchiors et al. (2000), Melchiors (2003) and Deshpande et al. (2003) for continuous review models) it is assumed that it is possible to discriminate among the customer classes by introducing a rationing policy. A rationing policy means that low priority customers are denied access to the inventory when the on-hand level is critically low. Thus, this policy may imply no access to the inventory even if there are actually items on inventory. 
Of course, if the item in question is extremely vital and the potential customer is a nuclear power plant, oil refinery or the like where an unfulfilled demand might imply a total stop of production with severe societal consequences, it is very sensible to apply such a rationing policy. Also, in some industries, e.g. in the airline industry, such procedures of deliberately denying access to an item are well accepted by customers. However, in other settings it may be less obvious to apply this rationing policy. If there is a substantial self-service element in the handling of an order request applying this policy maybe impossible or maybe even morally problematic if the low priority customer class is somewhat "sensitive". Instead of introducing a strict rationing policy, we believe that it would be more sensible to undertake customer relationship activities, such as persuading the customers to inform about their needs well in advance (maybe combined with enabling them to order via the Internet) and furthermore guarantee the customers that they will receive some degree of prioritized service. Then it will seem more reasonable to deny the fulfilment of an order request, even if there are items on stock, because now these items are reserved for customers who have submitted their requests well in advance. Hence, by combining elements of the sources of inspiration mentioned above, our aim is to study implementations of discrimination policies in the context of advance demand information.

In this paper we study the setting outlined in the beginning of this section. We assume that, from the perspective of the inventory system, the demand lead-times of the customers of Class 2 are random. We also assume that the inventory system is controlled by a base stock system (thus assuming negligible fixed replenishment costs), where all replenishments are issued instantaneously upon the receipt of an order. The aim is to find a good base stock policy giving both customer classes adequate service. The service measure that we will use is the order fill rate service (specified for each customer class), which is the fraction of orders where the customers get $100 \%$ fulfilment of their order requests at the delivery date (i.e. instantaneous fulfilment in the case of Class 1 customers). One of the issues that we will explore is how much priority should be given to the Class 2 customers. One option is to let the order be immediately reserved (or tagged, as we sometimes call it in the following) in the inventory system. This means that these items cannot be assigned to other customers whose requests arrive later. This is denoted complete reservation by Marklund (2006). Another option is to reserve the order only after a certain time delay. We then 
denote it a partial reservation policy. This may be a sensible approach if the demand lead-time of the customer is long, and thus keeping a certain amount free helps to provide adequate service also to the customers visting the retail outlet physically.

We study three ways of implementing a partial reservation policy, denoted Type 1, 2 and 3, respectively. Roughly speaking, the difference between Type 1 and 2 is that under the first policy, the order is only tagged in the inventory system $r$ time units (where $r$ is a policy variable) after the request has been registered, while according to the second policy, the order is only tagged in the inventory system $d$ time units (where $d$ is a policy variable) before it is needed. In the first case, one could interpret the request date as the anchor point, whereas in the second case, the need date is regarded as the anchor point for deciding when to reserve the order. Finally, according to the Type 3 reservation policy, the time between receiving and tagging an order depends on the actual demand lead-time and is proportional to it.

In Section 2 we will specify in more detail the mathematical assumption behind the inventory system. As some of the mathematical expressions are a bit involved, we will start out in Section 3 by deriving the order fill rates in the case of a single customer class, all customers of this class having positive (but random) demand lead-times. However, this is not only due to pedagogical reasons, but also because we find that this case provides insights in itself. Then in Section 4 we will show how to extend the mathematical expressions, derived in the previous section, to the case of the two customer classes. In Section 5 we will present the results of our numerical investigations. Finally, Section 6 contains some concluding remarks.

\section{The mathematical model}

We assume that the arrival processes of the order requests of the two customer classes are independent Poisson processes with intensities $\lambda_{j}, j=1,2$. The non-negative random variable $Y$, with density function $f(y)$, represents the demand lead-time of customers of Class 2 . That is, if an order request is placed at time point $\tau$ and the customer has a demand lead-time $y$, then the customer wants to get his demand fulfilled at time point $\tau+y$. The positive integer-valued random variables $X_{j}, j=1,2$ represent the order size of customer requests of Class 1 and 2, respectively. It is assumed that $X_{1}$ and $X_{2}$ are independent. Furthermore, any successive generations of the 
random variables $Y, X_{1}$ and $X_{2}$ are assumed independent as well. As $Y$ is assumed stochastic, it is understood that the customer's preference for a specific demand lead-time (or his willingness ahead of time to express his demand realization time) is outside the control of the inventory system. In the special case where it is a constant, this might represent a scenario where for instance the customer is only given the option of choosing a delivery lead-time when ordering from the Internet.

The inventory is controlled by a base stock policy with parameter $S$ (a non-negative integer). All unfulfilled demand is backlogged. It is assumed that all replenishment, which has a constant lead-time $L$, is done at exactly the time point when an order request occurs. Throughout the paper we assume that $\mathrm{P}(Y<L)=1$, as any order with a demand lead-time longer than the replenishment lead-time is a "known" order which can be handled outside the inventory system and is therefore not interesting when focusing on inventory control.

We now describe how the order fill rates are derived. First, consider an order of Class 2 specified by the triple $\left(\tau, x_{2}, y\right)$ (where $\tau$ is the arrival time, $x_{2}$ is the order size and $y$ is the demand lead-time) which has a reservation time $\mathrm{g}(\tau, y)$ where $\tau \leq \mathrm{g}(\tau, y) \leq \tau+y$. This means that at time point $\tau+\mathrm{g}(\tau, y)$ the order will be tagged and cannot be used by any orders having a reservation time after this time point. The order is completely fulfilled at $\tau+y$ if at this time point, the inventory level minus the total volume of orders not yet fulfilled and reserved prior to time point $\mathrm{g}(\tau, y)$ is larger than or equal to $x_{2}$. As inventory control is a base stock policy with base stock level $S$ and all replenishment orders are issued exactly when the requests occur, all requests received prior to time point $\tau+y-L$ are fulfilled and resupplied at the inventory at time point $\tau+y$. Therefore, the issue about complete fulfilment of an order of Class 2 can be summarized as the following:

Statement 1. An order of Class 2 specified by the triple $\left(\tau, x_{2}, y\right)$ (where $\tau$ is the arrival time, $x_{2}$ is the order size and $y$ is the demand lead-time) that has a reservation time $\mathrm{g}(\tau, y)$ where $\tau \leq \mathrm{g}(\tau, y) \leq$ $\tau+y$ is completely fulfilled at time point $\tau+y$ if $S$ minus $\{$ the total volume of orders received in the time interval $[\tau+y-L, \tau+\mathrm{g}(\tau, y))$ and tagged prior to time point $\tau+\mathrm{g}(\tau, y)\}$ is larger than or equal to $x_{2}$.

This statement can be applied to Class 1 as well because orders of Class 1 can similarly be characterized by a triple $\left(\tau, x_{1}, y\right)$. However, here $y=0$ and therefore $\mathrm{g}(\tau, y)=\tau$; that is, the order 
is reserved and attempted to be fulfilled immediately upon receipt. Therefore, we can also state the following:

Statement 2. An order of Class 1 specified by the triple $\left(\tau, x_{1}\right)$ (where $\tau$ is the arrival time and $x_{1}$ is the order size) is completely fulfilled at time point $\tau$ if $S$ minus the total volume of orders received in the time interval $[\tau-L, \tau)$ and tagged prior to time point $\tau\}$ is larger than or equal to $x_{1}$.

To complete the description of the inventory system: if an order cannot be completely fulfilled, the whole order is put on a waiting list. When receiving incoming replenishment orders, the waiting list is always attempted to be cleared and any waiting orders are prioritized according to the time of reservation.

We consider three reservation policies, called Type 1, 2 and 3, respectively. The Type 1 reservation policy is specified by a parameter $r$ where $0 \leq r \leq L$. This means that the aforementioned $\mathrm{g}(\tau, y)$ is specified as $\mathrm{g}(\tau, y)=\tau+\min (y, r)$. The parameter $r$ could be interpreted as the administrative delay that elapses before the order is reserved in the inventory system. If $y$ is small, $y \leq r$, then the order is never reserved. A special case of this reservation policy, with a constant demand lead-time, is studied in a slightly different context in Du and Larsen (2008) where it is denoted "Postpone". The Type 2 reservation policy is specified by a parameter $d$ where $0 \leq d \leq L$. Here $\mathrm{g}(\tau, y)=\tau+\max (y-d, 0)$. The logic behind the policy is to prioritize orders according to the customers' actual needs. If $y$ is small, $y \leq d$, then the order is reserved immediately when receiving the request. Finally, the Type 3 reservation policy is characterized by a parameter $\alpha$ where $0 \leq \alpha \leq 1$ and $\mathrm{g}(\tau, y)=\tau+\alpha y$. If $\alpha$ is chosen in between 0 and 1 , an order is always reserved prior to its need date and it is never reserved immediately when receiving the request. In this way, Type 3 is different from Types 1 and 2. This is also apparent from the fact that some of the derivations related to the Type 3 policy are handled differently than the derivations of the two other policy types. Note that when $r=0, d=L$ and $\alpha=0$, the reservation policies coincide and become the complete reservation policy, according to the definition in Marklund (2006). If $r=L, d=0$ and $\alpha=1$, the reservation policies also coincide and can be characterized as a "no reservation" case, analyzed in Marklund (2006) as well. 


\section{Derivations of order fill rates in the case of one customer class}

Here we consider an inventory system only serving customers of Class 2 . This case is interesting in itself because when $Y$ is a random variable, the sequence of order arrivals is not necessarily equal to the sequence of demand realizations. Therefore, the partial reservation policies have significance. Furthermore, when considering the case of the two customer classes, it is easier to see how the various formulas are generalized from the case of only one customer class. For simplicity, we will obviously skip index 2 in our derivations during this section. First, we will introduce some notations. Let $\operatorname{Po}(m)$ be a Poisson distributed random variable with mean $m$. For $a \leq b$, let $\mathrm{D}[a, b)$ denote the total demand of all requests received in the time interval $[a, b)$ (if $a>b$, we define $\mathrm{D}[a, b) \equiv 0)$. Per definition, $\mathrm{D}[a, b)=\sum_{i=1}^{\mathrm{Po}(\lambda(b-a))} X_{i}$ where $X_{i}, i=1,2, \ldots$ are independent random variables having the same distribution as $X$. For $0 \leq b \leq c \leq L$, let the random variable $N(b, c)$ denote the number of order requests received in the time interval $[0, b)$ with a demand realization (or need date) before time point $c$. Using the properties of a Poisson process, we can show that $N(b, c)$ is Poisson distributed with mean $\lambda\left[b \mathrm{P}(Y \leq c-b)+\int_{c-b}^{c}(c-y) f(y) \mathrm{d} y\right]$, see Appendix $A$.

\subsection{Partial reservation policy Type 1}

As derived in Appendix $B$, the order fill rate (specified as a function of the base stock level $S$ and the reservation policy variable $r$ ) is

$$
\begin{aligned}
\operatorname{OFR}(S, r) & =\int_{0}^{L} \mathrm{P}\left(X+\mathrm{D}\left[\tau+y-L, \tau-(r-y)^{+}\right)+\sum_{i=1}^{N(r, r)} X_{i} \leq S\right) f(y) \mathrm{d} y \\
& =\int_{0}^{L} \mathrm{P}\left(\begin{array}{c}
\operatorname{Po}\left(\lambda \left[L-y-(r-y)^{+}\right.\right. \\
\left.\left.+\int_{0}^{r}(r-x) f(x) \mathrm{d} x\right]\right) \\
\sum_{i=1} X_{i} \leq S
\end{array}\right) f(y) \mathrm{d} y
\end{aligned}
$$

If we assume that $r=L$, which means that there is no reservation, then we get

$$
\operatorname{OFR}(S, r)=\mathrm{P}\left(X+\sum_{i=1}^{\mathrm{Po}(\lambda(L-\mathrm{E}[Y]))} X_{i} \leq S\right)
$$


This tells us that when there is no reservation, we quite obviously do not care about the probability distribution of $Y$; only the mean value matters. This is also shown in Hariharan and Zipkin (1995) and it is actually a realization of Palm's Theorem, see Palm (1938) as well as, for instance, Zipkin (2000), pp. 247. If we assume that $r=0$, which means complete reservation, then we get

$$
\operatorname{OFR}(S, r)=\int_{0}^{L} \mathrm{P}\left(X+\sum_{i=1}^{\mathrm{Po}(\lambda(L-y))} X_{i} \leq S\right) f(y) \mathrm{d} y
$$

If we assume that the random variable $Y$ is a constant $y$, then we get

$$
\operatorname{OFR}(S, r)=\mathrm{P}\left(X+\sum_{i=1}^{\operatorname{Po}(\lambda(L-y))} X_{i} \leq S\right)
$$

Obviously, when all orders have the same demand lead-time, any reservation is uninteresting as the need sequence and the request sequence are the same.

\subsection{Partial reservation policy Type 2}

Here the order fill rate (specified as a function of the base stock level $S$ and the reservation policy variable $d$ ) is derived as, see the Appendix $C$.

$$
\begin{aligned}
\operatorname{OFR}(S, d) & =\int_{0}^{L} \mathrm{P}\left(X+\mathrm{D}[\tau+y-L, \tau+\max \{y, d\}-L)+\sum_{i=1}^{N(L-d, L)} X_{i} \leq S\right) f(y) \mathrm{d} y \\
& =\int_{0}^{L} \mathrm{P}\left(\begin{array}{c}
\operatorname{Po}(\lambda[L-\min (y, d) \\
\left.\left.+\int_{d}^{L}(d-x) f(x) \mathrm{d} x\right]\right) \\
+\sum_{i=1} X_{i} \leq S
\end{array}\right) f(y) \mathrm{d} y
\end{aligned}
$$

As a point of validation, note that when $d=0$ (no reservation), then $\operatorname{OFR}(S, d)$ is identical to the right hand side of (2) and when $d=L$ (complete reservation), then $\operatorname{OFR}(S, d)$ is identical to the right hand side of (3). Also, when $Y$ is a constant $y$, we get $\operatorname{OFR}(S, d)$ to be equal to the right hand side of (4). 


\subsection{Partial reservation policy Type 3}

Consider the time interval $[0, c)$ and let the random variable $M(c, \alpha)$ denote all customer requests received in this interval that under reservation policy Type 3 will be reserved prior to time point $c$. In Appendix $D$, we show that when $\alpha>0, M(c, \alpha)$ is Poisson distributed with mean $\lambda\left[c \mathrm{P}\left(Y \leq \frac{c}{\alpha}\right)-\alpha \int_{0}^{\frac{c}{\alpha}} y f(y) \mathrm{d} y\right]$. From Appendix $E$, we get that the order fill rate (specified as a function of the reservation parameter $\alpha$ and the base stock level $S$ ) is

$$
\operatorname{OFR}(S, \alpha)=\int_{0}^{L} \mathrm{P}\left(X+\sum_{i=1}^{\operatorname{Po}(\lambda[L-(1-\alpha) y-\alpha \mathrm{E}[Y]])} X_{i} \leq S\right) f(y) \mathrm{d} y
$$

As a point of validation, note that when $\alpha=1$ (no reservation), then $\operatorname{OFR}(S, \alpha)$ is identical to the right hand side of (2) and when $\alpha=0$ (complete reservation), then $\operatorname{OFR}(S, \alpha)$ is identical to the right hand side of (3). Also, when $Y$ is a constant $y$, we get $\operatorname{OFR}(S, \alpha)$ to be equal to the right hand side of (4).

\subsection{Comparisons of the reservation policies}

First of all one could ask: If there is only one single (homogenous) customer class, is it at all meaningful to make reservation of advance orders? Based on numerical studies, and proofs of simplified cases, our answer is no. A reservation policy causes some inefficiency as at a specific time one can simultaneously have positive on-hand inventory as well as a positive back-log. This is because some orders (not ready to be served yet) have already blocked the inventory system preventing other orders with shorter demand lead-times to access it. The next question one could ask is how a meaningful comparison between the three reservation policies can be made. Here we compare the expected tag times of the policies. We define a tag time as the interval between the time when the order is reserved and its need date. For Type 1 the expected tag time is $\int_{r}^{L}(y-$ $r) f(y) \mathrm{d} y$ while for Type 2 it is $\int_{0}^{d} y f(y) \mathrm{d} y+d \mathrm{P}(Y \geq d)$. Therefore, when comparing the Type 1 and the Type 2 reservation policies, $r$ and $d$ should be chosen such that

$$
\int_{r}^{L}(y-r) f(y) \mathrm{d} y=\int_{0}^{d} y f(y) \mathrm{d} y+d \mathrm{P}(Y \geq d)
$$


For Type 3 the expected tag time is $(1-\alpha) \mathrm{E}[Y]$. Therefore, when comparing the Type 1 and Type 3 reservation policies, $r$ and $\alpha$ should be chosen such that

$$
\int_{r}^{L}(y-r) f(y) \mathrm{d} y=(1-\alpha) \mathrm{E}[Y]
$$

Later in Section 5, the significance of these equations will be shown.

\section{Derivations of order fill rates in the case of two customer classes}

First we consider a specific Class 2 customer who arrives at time point $\tau$. From Statement 1 in Section 2 we know that for any demand lead-time $y$ and any of the reservation policies, all Class 1 customers arriving in the time interval $[\tau+y-L, \tau+\mathrm{g}(\tau, y))$ are served ahead of this order. Therefore, in the two customer classes case, the formulas (1), (5) and (6) should be adapted to

$$
\begin{aligned}
& \text { OFR }_{2}(S, r)=\int_{0}^{L} \mathrm{P}\left(\begin{array}{cl}
\begin{array}{c}
\operatorname{Po}\left(\lambda_{1}[L-y\right. \\
+\min (r, y)])
\end{array} & \begin{array}{l}
\operatorname{Po}\left(\lambda _ { 2 } \left[L-y-(r-y)^{+}\right.\right. \\
\left.\left.+\int_{0}^{r}(r-x) f(x) \mathrm{d} x\right]\right)
\end{array} \\
X_{2}+\sum_{i=1}\left(X_{1}\right)_{i} & +\sum_{i=1}\left(X_{2}\right)_{i} \leq S
\end{array}\right) f(y) \mathrm{d} y \\
& \text { OFR }_{2}(S, d)=\int_{0}^{L} \mathrm{P}\left(\begin{array}{cl}
\operatorname{Po}\left(\lambda_{1}[L-y\right. & \begin{array}{c}
\operatorname{Po}\left(\lambda_{2}[L-\min (y, d)\right. \\
\left.\left.+(y-d)^{+}\right]\right)
\end{array} \\
\left.\left.X_{2}+\sum_{i=1}^{L}(d-x) f(x) \mathrm{d} x\right]\right) \\
\end{array} \quad \begin{array}{l}
\left.\int_{1}\right)_{i} \\
+\sum_{i=1}\left(X_{2}\right)_{i} \leq S
\end{array}\right) f(y) \mathrm{d} y
\end{aligned}
$$

and

$$
\text { OFR }_{2}(S, \alpha)=\int_{0}^{L} \mathrm{P}\left(X_{2}+\sum_{i=1}^{\operatorname{Po}\left(\lambda_{1}[L-(1-\alpha) y]\right)}\left(X_{1}\right)_{i}+\sum_{i=1}^{\operatorname{Po}\left(\lambda_{2}[L-(1-\alpha) y\right.}\left(X_{2}\right)_{i} \leq S\right) f(y) \mathrm{d} y
$$

It still holds that when $r=L, d=0$ or $\alpha=1$, then no knowledge about $Y$; except $\mathrm{E}[Y]$ matters when deciding the suitable values of $S$ and the parameter of the chosen reservation policy.

Now we consider the derivations of the order fill rates for a specific Class 1 customer who arrives at time point $\tau$ (in the following referred to as Current-order). Irrespective of which of 
the three reservation policies applies, all the requests of the Class 1 customer received in the time interval $[\tau-L, \tau)$ (which is Poisson distributed with mean $\lambda_{1} L$ ) are served before this order. Under the Type 1 reservation policy, all customers of Class 2 whose requests either occur in the time interval $[\tau-L, \tau-r)$ or in the time interval $[\tau-r, \tau)$ with a need date before time point $\tau$ will be served ahead of Current-order. According to previous derivations the total number of these Class 2 customers is Poisson distributed with mean $\lambda_{2}\left[L-r+\int_{0}^{r}(r-x) f(x) \mathrm{d} x\right]$. Therefore, from Statement 2 we get that

$$
\text { OFR }_{1}(S, r)=\mathrm{P}\left(X_{1}+\sum_{i=1}^{\operatorname{Po}\left(\lambda_{1} L\right)}\left(X_{1}\right)_{i}+\sum_{i=1}^{\left.\left.+\int_{0}^{r o}(r-2) f(x) \mathrm{d} x\right]\right)}\left(X_{2}\right)_{i} \leq S\right)
$$

Under the Type 2 reservation policy, all customers of Class 2 whose requests either occur in the time interval $[\tau-L, \tau+d-L)$ or in the time interval $[\tau+d-L, \tau)$ and with a need date before time point $\tau+d$ will be served ahead of Current-order. According to previous derivations, the total number of these Class 2 customers is Poisson distributed with mean $\lambda_{2}\left[L+\int_{d}^{L}(d-x) f(x) \mathrm{d} x\right]$. Therefore, from Statement 2 we get that

$$
O F R_{1}(S, d)=\mathrm{P}\left(X_{1}+\sum_{i=1}^{\operatorname{Po}\left(\lambda_{1} L\right)}+\int_{d}^{L}\left(X_{1}\right)_{i}+\sum_{i=1}^{\operatorname{Po}\left(\lambda_{2}[L\right.}\left(X_{2}\right)_{i} \leq S\right)
$$

Concerning the Type 3 reservation policy, we should note that all customers of Class 2 arriving in the time interval $[\tau-L, \tau)$ and tagged before time point $\tau$ will be served ahead of Current-order. As this number ( $c=L$ in specification of the random variable $M(c, \alpha)$ in Appendix $D$ ) is Poisson distributed with mean $\lambda_{2}(L-\alpha \mathrm{E}[Y])$, we get

$$
\text { OFR }_{1}(S, \alpha)=\operatorname{Pr}\left(X_{1}+\sum_{i=1}^{\operatorname{Po}\left(\lambda_{1} L\right)}\left(X_{1}\right)_{i}+\sum_{i=1}^{\operatorname{Po}\left(\lambda_{2}[L-\alpha \mathrm{E}[Y]]\right)}\left(X_{2}\right)_{i} \leq S\right)
$$

By comparing (12) with (13), we get that customers of Class 1 are indifferent between the Type 1 and 2 reservation policies when (7) holds, and when comparing (12) with (14), we get that 
customers of Class 1 are indifferent between the Type 1 and 3 reservation policies when (8) holds. Note that the probability distribution of $Y$ has impact on the right hand side of (12), (13), but only through its mean in (14).

\section{Numerical results}

We have limited ourselves to the case where $Y$ is uniformly distributed between 0 and $L$, and where all customer orders, irrespective of class, are unit sizes, that is $\mathrm{P}\left(X_{1}=1\right)=\mathrm{P}\left(X_{2}=1\right)=1$. In the absence of any specific knowledge about what the demand lead-times could be, we assume them to be uniform between 0 to $L$, which we believe is a neutral assessment about the demand lead-times of the customers of Class 2. As our source of inspiration is a retail setting, we believe that here the assumption about unit demands could be reasonable. The previously derived formulas can then be considerably simplified. First, the order fill rate of Class 1 is under each of the three reservation policies

$$
\begin{aligned}
& \operatorname{OFR}_{1}(S, r)=\sum_{j=0}^{S-1} \mathrm{e}^{-\lambda_{1} L-\lambda_{2}\left(L-r+\frac{r^{2}}{2 L}\right)} \cdot \frac{\left(\lambda_{1} L+\lambda_{2}\left(L-r+\frac{r^{2}}{2 L}\right)\right)^{j}}{j !} \\
& \operatorname{OFR}_{1}(S, d)=\sum_{j=0}^{S-1} \mathrm{e}^{-\lambda_{1} L-\lambda_{2}\left(d+\frac{L^{2}-d^{2}}{2 L}\right)} \cdot \frac{\left(\lambda_{1} L+\lambda_{2}\left(d+\frac{L^{2}-d^{2}}{2 L}\right)\right)^{j}}{j !} \\
& \operatorname{OFR}_{1}(S, \alpha)=\sum_{j=1}^{S-1} \mathrm{e}^{-\lambda_{1} L-\lambda_{2} L\left(1-\frac{\alpha}{2}\right)} \cdot \frac{\left(\lambda_{1} L+\lambda_{2} L\left(1-\frac{\alpha}{2}\right)\right)^{j}}{j !}
\end{aligned}
$$

By exploiting that for $0 \leq a \leq b$ and $j=0,1,2, \cdots: \int_{a}^{b} \mathrm{e}^{-t \frac{t^{j}}{j !}} \mathrm{d} t=\sum_{i=0}^{j} \mathrm{e}^{-a} \frac{t^{a}}{i !}-\sum_{i=0}^{j} \mathrm{e}^{-b \frac{t^{b}}{i !}}$, we get for Class 2

$$
\begin{aligned}
\operatorname{OFR}_{2}(S, r)= & \frac{1}{L\left(\lambda_{1}+\lambda_{2}\right)}\left\{\sum_{j=0}^{S-1}\left(r\left(\lambda_{1}+\lambda_{2}\right)+j-S\right) \mathrm{e}^{-\lambda_{1} L-\lambda_{2}\left(L-r+\frac{r^{2}}{2 L}\right)} \cdot \frac{\left(\lambda_{1} L+\lambda_{2}\left(L-r+\frac{r^{2}}{2 L}\right)\right)^{j}}{j !}\right. \\
& \left.+\sum_{j=0}^{S-1}(S-j) \mathrm{e}^{-\lambda_{1} r-\lambda_{2} \frac{r^{2}}{2 L}} \cdot \frac{\left(\lambda_{1} r+\lambda_{2} \frac{r^{2}}{2 L}\right)^{j}}{j !}\right\}
\end{aligned}
$$




$$
\begin{aligned}
\operatorname{OFR}_{2}(S, d)= & \frac{1}{L\left(\lambda_{1}+\lambda_{2}\right)}\left\{\sum_{j=0}^{S-1}\left((L-d)\left(\lambda_{1}+\lambda_{2}\right)+S-j\right) \mathrm{e}^{-\lambda_{1}(L-d)-\lambda_{2} \frac{L^{2}-d^{2}}{2 L}} \cdot \frac{\left(\lambda_{1}(L-d)+\lambda_{2} \frac{L^{2}-d^{2}}{2 L}\right)^{j}}{j !}\right. \\
& \left.-\sum_{j=0}^{S-1}(S-j) \mathrm{e}^{-\lambda_{1} L-\lambda_{2}\left(d+\frac{L^{2}-d^{2}}{2 L}\right)} \cdot \frac{\left(\lambda_{1} L+\lambda_{2}\left(d+\frac{L^{2}-d^{2}}{2 L}\right)\right)^{j}}{j !}\right\}
\end{aligned}
$$

and when $\alpha<1$

$$
\begin{aligned}
\operatorname{OFR}_{2}(S, \alpha)= & \frac{1}{L(1-\alpha)\left(\lambda_{1}+\lambda_{2}\right)} \sum_{j=1}^{S-1}(S-j)\left\{\mathrm{e}^{-\lambda_{1} \alpha L-\lambda_{2} \frac{\alpha L}{2}} \cdot \frac{\left(\lambda_{1} \alpha L-\lambda_{2} \frac{\alpha L}{2}\right)^{j}}{j !}\right. \\
& \left.-\mathrm{e}^{-\lambda_{1} L-\lambda_{2} L\left(1-\frac{\alpha}{2}\right)} \cdot \frac{\left(\lambda_{1} L+\lambda_{2} L\left(1-\frac{\alpha}{2}\right)\right)^{j}}{j !}\right\}
\end{aligned}
$$

For this special case, (7) can be rewritten to

$$
d=L-\sqrt{2 L r-r^{2}}
$$

and (8) can be rewritten to

$$
\alpha=\frac{2 L r-r^{2}}{L^{2}}
$$

In Table 1, we present a numerical study of the one customer class case. The tables are organized such that for all policies the top entry represents the situation with complete reservation and then by traversing down the level of reservation decrease until reaching in the bottom entry representing the situation with no reservation.

\section{Table 1a-c about here}

For a pre-specified minimum order fill rate of $90 \%$, we find in each entry the minimum value of the base stock parameter $S$ that secures this. We have also collected information about the average 
inventory level. As this is hard to express mathematically, it is found by using a simulation model, developed in Arena, see Kelton et al. (2007). As we move down in the tables, the base stock parameter can be adjusted upwards, implying a lower average inventory level. This illustrates the claim made earlier that when having a single homogenous customer class and, maybe more importantly, applying the same service measures to all customers of this class, then it does not make sense to adopt a reservation policy.

Now in Table 2, we present a numerical study of the two customer classes case where we compare the three reservation policies and, by using expressions (21) and (22), neutralize the effect of the fill rate performance on the customers of Class 1.

\section{Table 2 about here}

In order to better compare the three policies, we have kept $S$ fixed when varying the parameters $r, d$ and $\alpha$. We see that for comparable values of $r, d$ and $\alpha$ it is under the Type 2 reservation policy that the customers of Class 2 enjoy the best order fill rate and that the inventory level is lowest. Based on this example (and others not reported here), Type 2 therefore seems to be the best policy, followed by Type 3 and Type 1. It is maybe not so odd that Type 2 performs best because it seems very natural to base the timing of the reservation on the need date. Also, note that while $O F R_{2}(S, r)$ is decreasing as a function of $r$, which seems only natural, this is not the case for $\operatorname{OFR}_{2}(S, d)$ and $\operatorname{OFR}_{2}(S, \alpha)$ which are unimodal as functions of $d$ and $\alpha$, respectively. So for these two reservation policies, also in the presence of other customers, it seems as if Class 2 customers prefer partial reservation to complete reservation; thus reserving items in advance is not always of advantage to them. It is also apparent that allowing some delay between the request date and the time when the order is tagged makes it possible for a given value of $S$ (which is roughly a good estimate of a given inventory investment) to balance the service levels offered to each customer class better.

Having observed that the Type 2 reservation policy performs best compared to the others, we study in the following how the $d$ parameter should be set optimally. In Table 3 and 4, we examine how $d$ and $S$ should be set in order to minimize the average on-hand inventory with respect to a $90 \%$ target on the weighted average of the two order fill rates, where the weights are specified according to the demand rates. The summary of this investigation is presented in Table 4 while a 
more detailed presentation of the results concerning one of these cases is presented in Table 3 .

\section{Table 3-4 about here}

It turns out that one should always set the $d$ parameter equal to zero. That is, the customers of Class 2 should not be offered any privileges in terms of reserving their orders prior to their need dates. We could already foresee this result from the entries in Table $1 \mathbf{b}$ when the demand rate of Class 2 is relatively high. On the other hand, when the demand rate of Class 1 is relatively high, then of course much weight is placed on the order fill rate of this class, and therefore $d$ should also be zero here. We have noted the same phenomenon in other cases not reported here. As expected, when the fraction of Class 2 orders increases, the minimum average inventory level decreases, most significantly when going from $\left(\lambda_{1}, \lambda_{2}\right)=(1,1)$ to $\left(\lambda_{1}, \lambda_{2}\right)=(0.5,1.5)$. Of course, we should note here that because of the integer requirement on $S$, the weighted service level is well above $90 \%$ for the case $\left(\lambda_{1}, \lambda_{2}\right)=(1,1)$. One way of forcing a positive value of $d$ to be optimal is by letting the weights of the two order fill rates deviate from the demand rates by using weights $\left(w_{1}, w_{2}\right)$ with $w_{1}+w_{2}=1$ and $w_{2} \geq \lambda_{2} /\left(\lambda_{1}+\lambda_{2}\right)$. This could be justified by assigning higher importance to the customers of Class 2, maybe because they pay a higher price. So as an experiment, we used the same data set as in Table 3 to examine how much the weights should be changed before an optimal solution with $d>0$ is chosen. It turns out that when $w_{2}$ is 0.6 , then the solution shifts from $(S, d)=(18,0)$ to $(S, d)=(17,1)$, giving Class 1 an order fill rate of $84.00 \%$, Class 2 an order fill rate of $94.04 \%$ while the average inventory is 5.1681. Thus, in this example a very moderate change of the weights from the fraction of demand rates makes the reservation policy advantageous.

\section{Concluding remarks}

We have studied an inventory model with two different customer classes and proposed three partial reservation policies for handling advance orders. For the case of a compound Poisson process, we have derived order fill rate service measures. For the case of unit size demands and the demand lead-times uniformly distributed, our numerical results indicate that the Type 2 reservation policy is dominant. This is maybe not so surprising because this reservation policy tends to time 
the point of tagging the order using the need date as an anchoring point. We conjecture that this result holds in more general cases as well. Our numerical studies also indicate that if the importance of the two customer classes is weighted according to their demand rates, then it is not a good idea to reserve orders in advance. Of course the manager of the inventory system may do this anyway in anticipation of future increased demand from Class 2 customers because of the reservation policy. So unless this is the case or there is a difference in the importance of the two customer classes (maybe because different prices are charged), managers should be careful to provide advance reservation of orders. We also consider extending the model to other service measures, such as the volume fill rate. However, here we should keep in mind than in many cases (e.g. unit size demand or geometrically distributed order sizes) the volume fill rate is actually identical to the order fill rate, see Larsen and Thorstenson (2008). Similarly, we are considering how the derived formulas can be extended when the arrival patterns are not Poisson processes, like a compound renewal (Erlang) process. However, this might be quite a challenge and we conjecture that it is only possible to derive tractable computational expressions if we let the demand lead-time be a constant, as in Du and Larsen (2008).

\section{Acknowledgement}

This work is supported by grant no. 275-07-0094 from the Danish Social Science Research Council.

\section{References}

Chen, F., 2001. Market segmentation, advanced demand information, and supply chain performance. Manufacturing \& Service Operations Management 3 (1), 53-67.

Dekker, R., Hill, R., Kleijn, M., Teunter, R., 2002. On the $(s-1, s)$ lost sales inventory model with priority demand classes. Naval Research Logistics 49 (6), 593-610.

Dekker, R., Kleijn, M., de Rooij, P., 1998. A spare parts stocking policy based on equipment criticality. International Journal of Production Economics 56-57, 67-77. 
Deshpande, V., Cohen, M., Donohue, K., 2003. A threshold inventory rationing policy for servicedifferentiated demand classes. Management Science 49 (6), 683-703.

Du, B., Larsen, C., 2008. Base stock policies with degraded service to larger orders. CORAL Working Paper, Department of Business Studies, Aarhus School of Business, Aarhus University, Denmark, http://www.hha.dk/bs/wp/log/L_2008_07.pdf.

Evans, R., 1968. Sales and restocking in a single item inventory system. Management Science $14(7), 463-472$.

Frank, K., Zhang, R., Duenyas, I., 2003. Optimal policies for inventory systems with priority demand classes. Operations Research 51 (6), 993-1002.

Gallego, G., Özer, Ö., 2001. Integrating replenishment decisions with advance demand information. Management Science 47 (10), 1344-1360.

Gayon, J.-P., Benjaafar, S., de Véricourt, F., 2009. Using imperfect advance demand information in production-inventory systems with multiple customer classes. Manufacturing \& Service Operations Management 11 (1), 128-143.

Hariharan, R., Zipkin, P., 1995. Customer-order information, leadtimes, and inventories. Management Science 41 (10), 1599-1607.

Kaplan, A., 1969. Stock rationing. Management Science 15 (5), 260-267.

Kelton, W., Sadowski, R., Sturrock, D., 2007. Simulation with ARENA, 4th Edition. McGraw Hill: Boston.

Kunnumkal, S., Topaloglu, H., 2008. Price discounts in exchange for reduced customer demand variability and applications to advance demand information acquisition. International Journal of Production Economics 111, 543-561.

Larsen, C., Thorstenson, A., 2008. A comparison between the order and the volume fill rate for a base-stock inventory control system under a compound renewal demand process. Journal of the Operational Research Society 59, 798-804. 
Lu, Y., Song, J.-S., Yao, D. D., 2003. Order fill rate, leadtime variability, and advance demand information in an assemble-to-order system. Operations Research 51 (2), 292-308.

Marklund, J., 2006. Controlling inventories in divergent supply chains with advance-order information. Operations Research 54 (5), 988-1010.

Melchiors, P., 2003. Restricted time remembering policies for the inventory rationing problem. International Journal of Production Economics 81-82, 461-468.

Melchiors, P., Dekker, R., Kleijn, M., 2000. Inventory rationing in an $(s, Q)$ inventory model with two demand classes and lost sales. Journal of the Operational Research Society 51 (1), 111-122.

Nahmias, N., Demmy, W., 1981. Operating characteristics of an inventory system with rationing. Management Science 27 (11), 1236-1245.

Palm, C., 1938. Analysis of the erlang traffic formula for busy signal arrangements. Ericson Technics 4, 204-212.

Papier, F., Thonemann, U. W., 2009. Capacity rationing in stochastic rental systems with advance demand information. Operations Research, Articles in Advance.

Silver, E., Pyke, D., Peterson, R., 1998. Inventory Management and Production Planning and Scheduling, Third Edition. John Wiley \& Sons: New York, NY.

Simchi-Levi, D., Kaminsky, P., Simchi-Levi, E., 2008. Designing \& Managing the Supply Chain, 3rd Edition. McGraw-Hill/Irwin: Boston.

Tan, T., 2008. Using imperfect advance demand information in forecasting. IMA Journal of Management Mathematics 19, 163-173.

Tan, T., Güllü, R., Erkip, N., 2009. Using imperfect advance demand information in ordering and rationing decisions. International Journal of Production Economics 121, 665-677.

Tempelmeier, H., 2006. Supply chain inventory optimization with two customer classes in discrete time. European Journal of Operations Research 174, 600-621. 
Topkis, D., 1968. Optimal ordering and rationing policies in a nonstationary dynamic inventory model with $n$ demand classes. Management Science 15 (3), 160-176.

Wang, T., Toktay, B., 2008. Inventory management with advance demand information and flexible delivery. Management Science 54 (4), 716-732.

Zipkin, P., 2000. Foundations of Inventory Management. McGraw-Hill: Boston.

\section{Appendices}

\section{Appendix A: Proof that $N(b, c)$ is Poisson distributed}

First, consider a simplified situation where $Y$ can only attain three different values: $y_{1}, y_{2}$ and $y_{3}$ with probabilities $p_{1}, p_{2}$ and $p_{3}$, respectively. Assume that $0 \leq y_{1} \leq c-b \leq y_{2} \leq c \leq y_{3} \leq L$. Using the theory about splitting a Poisson process, the requests of customers all having a demand lead-time $y_{j}$ arrive according to a Poisson process with intensity $\lambda p_{j}, j=1,2,3$ and all three Poisson processes are independent. All the customer requests with a demand lead-time $y_{1}$ arriving in the time interval $[0, b)$ will have a need date before $c$. All the customer requests with a demand lead-time $y_{2}$ arriving in the time interval $\left[0, c-y_{2}\right)$ will have a need date before $c$ (and the requests arriving in the interval $\left[c-y_{2}, b\right)$ will have a need date time point at or after $c$ ). Finally, no requests with a demand lead-time $y_{3}$ arriving in the time interval $[0, b)$ will have a need date time before $c$. From the theory about merging independent Poisson processes we therefore get that for this simplified situation $N(b, c)$ is Poisson distributed with mean $\lambda\left[b p_{1}+\left(c-y_{2}\right) p_{2}\right]$. For any discrete distribution of $Y$, this argument can be generalized to the case that $N(b, c)$ is Poisson distributed with mean $\lambda\left[b \mathrm{P}(Y \leq c-b)+\sum_{i: b<y_{i} \leq c}\left(c-y_{i}\right) \mathrm{P}\left(Y=y_{i}\right)\right]$. Strictly speaking, when $Y$ is a continuously distributed, in order to make a rigorous proof, we have to present our arguments through a differential equation:

Focusing on an infinitesimal increment in time by $\Delta$, we get that

$$
\mathrm{P}(N(b, c)=x)=\left\{\begin{array}{cc}
\lambda \Delta \mathrm{P}(Y \leq c-\Delta) \mathrm{P}(N(b-\Delta, c-\Delta)=x-1) & x \geq 1 \\
+(1-\lambda \Delta \mathrm{P}(Y \leq c-\Delta)) \mathrm{P}(N(b-\Delta, c-\Delta)=x) & \\
(1-\lambda \Delta \mathrm{P}(Y \leq c-\Delta)) \mathrm{P}(N(b-\Delta, c-\Delta)=0) & x=0
\end{array}\right.
$$


By collecting terms and letting $\Delta$ approach zero, we get

$$
\begin{aligned}
\frac{\delta \mathrm{P}(N(b, c)=x)}{\delta b}+ & \frac{\delta \mathrm{P}(N(b, c)=x)}{\delta c} \\
& = \begin{cases}\lambda \mathrm{P}(Y \leq c)[\mathrm{P}(N(b, c)=x-1)-\mathrm{P}(N(b, c)=x)] & x \geq 1 \\
-\lambda \mathrm{P}(Y \leq c) \mathrm{P}(N(b, c)=0) & x=0\end{cases}
\end{aligned}
$$

We can now verify that when $N(b, c)$ is Poisson distributed with mean $\lambda[b \mathrm{P}(Y \leq c-b)+$ $\int_{c-b}^{c}(c-y) f(y) \mathrm{d} y$ ], its probabilities solve the differential equation above. Strictly speaking, we only needed to provide proof for the most general case. However, for pedagogical reasons, we have also provided a simpler proof for the case where $Y$ is a discrete random variable.

\section{Appendix B: Derivation of OFR under Type 1 reservation}

Consider a customer arriving at time point $\tau$ with demand lead-time $y$ and denote it Currentorder. By distinguishing whether $y>r$ or $y \leq r$, we have identified which request will be served prior to Current-order, see the legend of Figure 1. These findings can be summarized as follows: all orders received in the time interval $\left[\tau+y-L, \tau-(r-y)^{+}\right)$will be served ahead of Current-order while the orders received in the time interval $\left[\tau-(r-y)^{+}, \tau+\min (r, y)\right)$ with a need date before time point $\tau+\min (r, y)$ will be served ahead of Current-order. The latter number $\left(0=\tau-(r-y)^{+}, b=\right.$ $\left.c=\min (r, y)+(r-y)^{+}=r\right)$ is specified by the random variable $N(r, r)$. Because we do not know the value of $y$ and the size of Current-order in advance, the order fill rate is as specified in (1).

\section{Figure 1 about here}

\section{Appendix C: Derivation of OFR under Type 2 reservation}

Consider a customer arriving at time point $\tau$ with demand lead-time $y$ and denote it Currentorder. Distinguishing between the cases $y>d$ and $y \leq d$ and summarizing the findings of Figure 2 , we get that all orders received in the time interval $[\tau+y-L, \tau+\max (y, d)-L)$ will be served ahead of Current-order while the orders received in the time interval $\left[\tau+\max (y, d)-L, \tau+(y-d)^{+}\right)$ with a demand need date before time point $\tau+\max (y, d)$ will be served ahead of Current-order. The latter number $\left(0=\tau+\max (y, d)-L, b=\tau+(y-d)^{+}-\tau-\max (y, d)+L=L-d\right.$ and 
$c=\tau+\max (y, d)-\tau-\max (y, d)+L=L)$ is specified by the random variable $N(L-d, L)$. Because we do not know the value of $y$ and the size of Current-order in advance, the order fill rate is as specified in (5).

\section{Figure 2 about here}

\section{Appendix D: Proof that $M(c, \alpha)$ is Poisson distributed}

Assume first that $Y$ is a discrete random variable. Using the same argumentation as in Appendix $A$ about first splitting and merging independent Poisson processes, we get that $M(c, \alpha)$ is Poisson distributed with mean $\lambda\left[c \mathrm{P}(Y \leq c / \alpha)-\alpha \sum_{i: y_{i} \leq c / \alpha} y_{i} \mathrm{P}\left(Y=y_{i}\right)\right]$. For the case where $Y$ is a continuous random variable, we have to provide rigorous arguments based on differential equations. By focusing on a small time increment $\Delta$, we get

$$
\mathrm{P}(M(c, \alpha)=x)=\left\{\begin{array}{cc}
\lambda \Delta \mathrm{P}(Y \leq(c-\Delta) / \alpha) \mathrm{P}(M(c-\Delta, \alpha)=x-1)+ & x \geq 1 \\
(1-\lambda \Delta \mathrm{P}(Y \leq(c-\Delta) / \alpha)) \mathrm{P}(M(c-\Delta, \alpha)=x) & \\
(1-\lambda \Delta \mathrm{P}(Y \leq(c-\Delta) / \alpha)) \mathrm{P}(M(c-\Delta, \alpha)=0) & x=0
\end{array}\right.
$$

By collecting terms and letting $\Delta$ approach zero, we get

$$
\frac{\delta \mathrm{P}(M(c, \alpha)=x)}{\delta c}= \begin{cases}\lambda \mathrm{P}(Y \leq c / \alpha) \mathrm{P}(M(c, \alpha)=x-1)-\mathrm{P}(M(c, \alpha)=x) & x \geq 1 \\ -\lambda \mathrm{P}(Y \leq c / \alpha)) \mathrm{P}(M(c-\Delta, \alpha)=0) & x=0\end{cases}
$$

From this we derive that $M(c, \alpha)$ is Poisson distributed with mean $\lambda \int_{0}^{c} \mathrm{P}(Y \leq y / \alpha) \mathrm{d} y$. To ensure completeness of the specification, note that if $\alpha=0$, then obviously $M(c, \alpha)$ is Poisson distributed with mean $\lambda c$. In order to show the equivalence to the discrete case, note that

$$
\int_{0}^{c} \mathrm{P}\left(Y \leq \frac{c}{\alpha}\right) \mathrm{d} y=c \mathrm{P}\left(Y \leq \frac{c}{\alpha}\right)-\alpha \int_{0}^{\frac{c}{\alpha}} y f(y) \mathrm{d} y
$$




\section{Appendix E: Derivation of OFR under Type 3 reservation}

Consider a customer arriving at time point $\tau$ with demand lead-time $y$ and denote it Currentorder. As it will be tagged at time point $\tau+\alpha y$, we need to focus on the number of requests received in the interval $[\tau+y-L, \tau+\alpha y)$ that are reserved before time point $\tau+\alpha y$. This number is specified by the random variable $M(L-(1-\alpha) y, \alpha)(0=\tau+y-L$ and $c=\tau+\alpha y-\tau+y-L=L-(1-\alpha) y)$. Because we assume that $\mathrm{P}(Y \leq L)=1$, it holds that $c / \alpha \geq L$. Therefore, we get that the number of requests received in the interval $[\tau+y-L, \tau+\alpha y)$ that are reserved before Current-order is Poisson distributed with mean $\lambda[L-(1-\alpha) y-\alpha \mathrm{E}[Y])$. Because we do not know the value of $y$ and the size of Current-order in advance, the order fill rate is as specified in (6). 


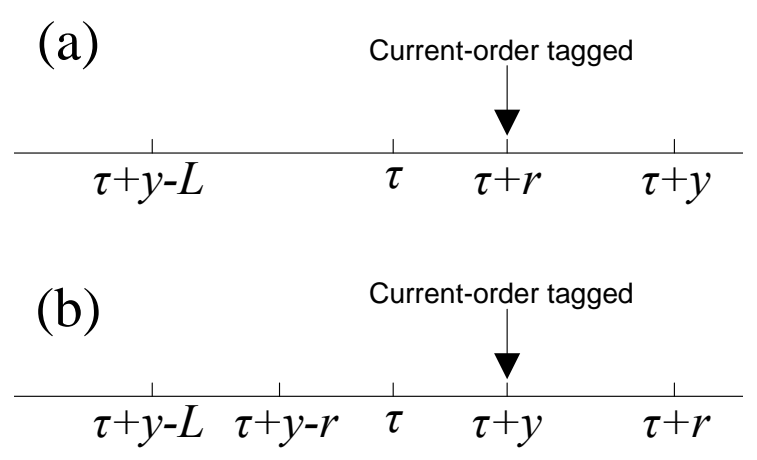

Figure 1: (a) All requests received in the time interval $[\tau+y-L, \tau)$ will be served ahead of Current-order. All requests received in the time interval $[\tau, \tau+r)$ with a demand realization before time point $\tau+r$ will be served ahead of Currentorder. (b) All requests received in the time interval $[\tau+y-L, \tau+y-r$ ) will be served ahead of Current-order. All requests received in the time interval $[\tau+y-r, \tau+y)$ with a demand realization before time point $\tau+y$ will be served ahead of Current-order.
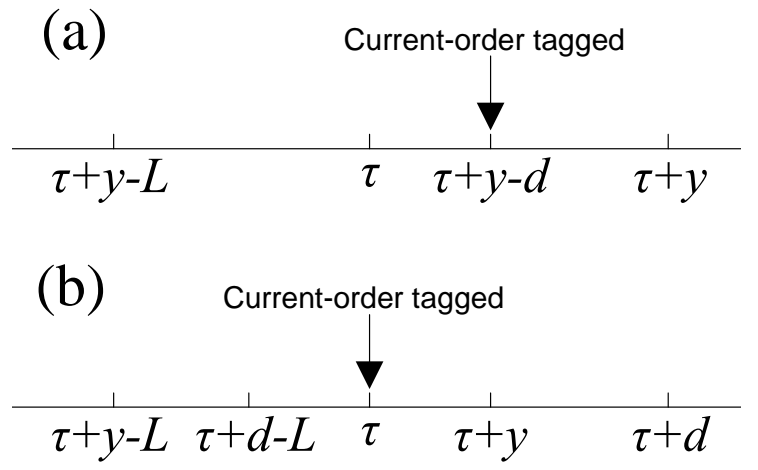

Figure 2: (a) All requests received in the time interval $[\tau+y-L, \tau+y-d)$ with a demand realization before time point $\tau+y$ will be served ahead of Current-order. (b) All orders received in the time interval $[\tau+y-L, \tau+d-L$ ) will be served ahead of Current-order. All orders received in the time interval $[\tau+d-L, \tau)$ with a demand realization before time point $\tau+d$ will be served ahead of Current-order. 
Table 1: (a) The one customer class case. Reservation policy Type 1, $L=4$, unit demand, $Y$ uniform on 0 to $L$. Required minimum order fill rate is $90 \%$.

\begin{tabular}{c|c|c|c}
\hline$r$ & $S$ & $A v . I n v .($ Sim $)$ & OFR \\
\hline 0 & 9 & 5.1113 & $91.13 \%$ \\
1 & 9 & 5.0693 & $92.97 \%$ \\
2 & 8 & 4.0682 & $91.81 \%$ \\
3 & 8 & 4.0354 & $94.34 \%$ \\
4 & 8 & 4.0292 & $94.89 \%$ \\
\hline
\end{tabular}

Table 1: (b) The one customer class case. Reservation policy Type 2, $L=4$, unit demand, $Y$ uniform on 0 to $L$. Required minimum order fill rate is $90 \%$.

\begin{tabular}{c|c|c|c}
\hline$d$ & $S$ & $A v . I n v .(S$ im $)$ & OFR \\
\hline 4 & 9 & 5.1113 & $91.13 \%$ \\
3 & 9 & 5.0904 & $92.35 \%$ \\
2 & 8 & 4.0935 & $91.45 \%$ \\
1 & 8 & 4.0394 & $94.28 \%$ \\
0 & 8 & 4.0292 & $94.89 \%$ \\
\hline
\end{tabular}

Table 1: (c) The one customer class case. Reservation policy Type 3, $L=4$, unit demand, $Y$ uniform on 0 to $L$. Required minimum order fill rate is $90 \%$.

\begin{tabular}{c|c|c|c}
\hline$\alpha$ & $S$ & $A v$. Inv.(Sim) & OFR \\
\hline 0 & 9 & 5.1113 & $91.13 \%$ \\
0.25 & 9 & 5.0611 & $93.82 \%$ \\
0.5 & 8 & 4.0701 & $92.16 \%$ \\
0.75 & 8 & 4.0382 & $94.16 \%$ \\
1 & 8 & 4.0292 & $94.89 \%$ \\
\hline
\end{tabular}


Table 2: The two customer classes case with $L=4, \lambda_{1}=\lambda_{2}=1, Y$ uniform on $(0,4), X_{1} \equiv X_{2} \equiv 1$ and $S=10$. Three types of Partial Reservation Policy. All types have the same $O F R_{1}$.

\begin{tabular}{c|c|c|c|c|c|c|c|c|c}
\hline \multicolumn{3}{c|}{ Type 1 } & \multicolumn{3}{c|}{ Type 2 } & \multicolumn{3}{c|}{ Type 3 } & \\
\hline$r$ & $O F R_{2}$ & AOI & $d$ & $O F R_{2}$ & AOI & $\alpha$ & $O F R_{2}$ & AOI & $O F R_{1}$ \\
\hline 0 & 0.9468 & 4.2430 & 4 & 0.9468 & 4.2430 & 0 & 0.9468 & 4.2430 & 0.7166 \\
1 & 0.9265 & 4.1519 & 1.3542 & 0.9631 & 4.1269 & 0.4375 & 0.9504 & 4.1348 & 0.8176 \\
2 & 0.9226 & 4.1020 & 0.5359 & 0.9454 & 4.0854 & 0.75 & 0.9408 & 4.0891 & 0.8774 \\
3 & 0.9200 & 4.0803 & 0.1270 & 0.9244 & 4.0767 & 0.9375 & 0.9241 & 4.0770 & 0.9072 \\
4 & 0.9161 & 4.0761 & 0 & 0.9161 & 4.0761 & 1 & 0.9161 & 4.0761 & 0.9161 \\
\hline
\end{tabular}

Note: AOI denotes the Average On-hand Inventory.

Table 3: The two customer classes case with $L=8, \lambda_{1}=\lambda_{2}=1, Y$ uniform on $(0, L), X_{1} \equiv X_{2} \equiv 1$. For each value of $d, S^{*}(d)$ is the least value of $S$ where $\frac{\lambda_{1}}{\lambda_{1}+\lambda_{2}} O F R_{1}(S, d)+\frac{\lambda_{2}}{\lambda_{1}+\lambda_{2}} O F R_{2}(S, d) \geq 0.9$. Then the resulting consequence for the average on-hand inventory is evaluated in a simulation model. The optimal solution is therefore $d^{*}=0$ and $S^{*}=15$.

\begin{tabular}{c|c|c|c|c|c|c|c|c|c}
\hline$d$ & 0 & 1 & 2 & 3 & 4 & 5 & 6 & 7 & 8 \\
\hline$S^{*}(d)$ & 18 & 18 & 18 & 19 & 20 & 20 & 20 & 21 & 21 \\
Av.OFR(\%) & 93.70 & 92.65 & 91.00 & 92.04 & 93.10 & 91.62 & 90.46 & 92.87 & 92.67 \\
Av.Inv.(sim) & 6.0792 & 6.0972 & 6.1453 & 7.1314 & 8.1125 & 8.1463 & 8.1743 & 9.1213 & 9.1259 \\
\hline
\end{tabular}


Table 4: The two customer classes case with $L=4, Y$ uniform on $(0,4), X_{1} \equiv X_{2} \equiv 1$. For each combination of $\left(\lambda_{1}, \lambda_{2}\right), d^{*}$ and $S^{*}$ are found such that $\frac{\lambda_{1}}{\lambda_{1}+\lambda_{2}} O F R_{1}(S, d)+\frac{\lambda_{2}}{\lambda_{1}+\lambda_{2}} O F R_{2}(S, d) \geq 0.9$ while minimizing the average on-hand inventory.

\begin{tabular}{c|c|c|c|c|c}
\hline$\lambda_{1}$ & $\lambda_{2}$ & $d^{*}$ & $S^{*}$ & Av. OFR & Av.Inv.(sim) \\
\hline 1.5 & 0.5 & 0 & 20 & $92.35 \%$ & 6.1007 \\
1 & 1 & 0 & 18 & $93.70 \%$ & 6.0792 \\
0.5 & 1.5 & 0 & 15 & $91.65 \%$ & 5.1058 \\
\hline
\end{tabular}

